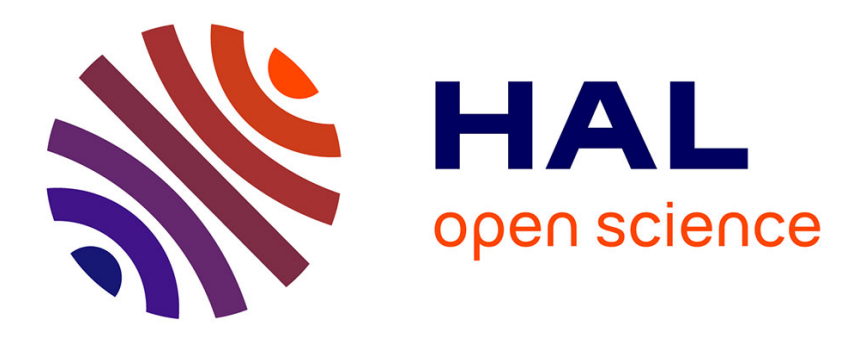

\title{
Les effets des politiques de Quantitative Easing sur le taux de change: Les enseignements de l'expérience américaine
}

M. Dupuy

\section{To cite this version:}

M. Dupuy. Les effets des politiques de Quantitative Easing sur le taux de change: Les enseignements de l'expérience américaine. 2013. hal-00780498

\section{HAL Id: hal-00780498 \\ https://hal.science/hal-00780498}

Preprint submitted on 24 Jan 2013

HAL is a multi-disciplinary open access archive for the deposit and dissemination of scientific research documents, whether they are published or not. The documents may come from teaching and research institutions in France or abroad, or from public or private research centers.
L'archive ouverte pluridisciplinaire HAL, est destinée au dépôt et à la diffusion de documents scientifiques de niveau recherche, publiés ou non, émanant des établissements d'enseignement et de recherche français ou étrangers, des laboratoires publics ou privés. 


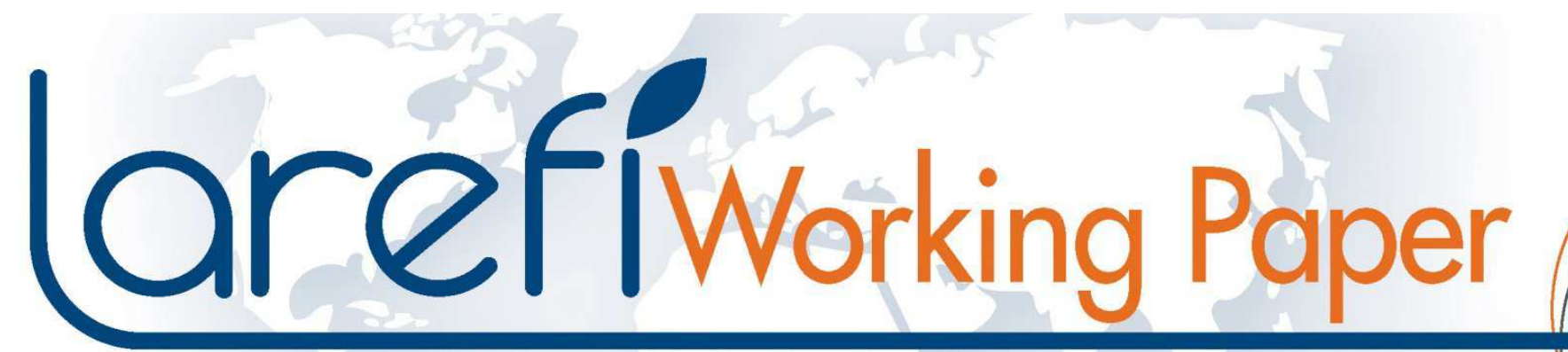

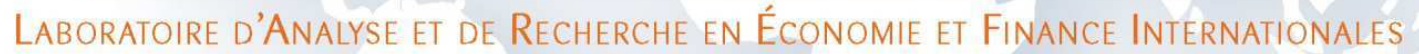

\section{Les effets des politiques de Quantitative Easing sur le taux de change :}

\section{Les enseignements de l'expérience américaine}

Michel Dupuy

LAREFI Working Paper N²013-02

Janvier 2013

http://lare-efi.u-bordeaux4.fr

LAREFI

Université Montesquieu-Bordeaux IV

Bâtiment Recherche Economie $-1^{\text {er }}$ étage

Avenue Léon Duguit - 33608 Pessac 


\section{LAREFI - LABORATOIRE D'ANALYSE ET DE RECHERCHE EN ECONOMIE ET FINANCES INTERNATIONALES}

\section{AUTHORS}

Michel Dupuy, LAREFI, Université Montesquieu Bordeaux IV and IFPRI Washington DC

\section{NOTICES}

LAREFI Working Papers contain preliminary material and research results. They have been peer reviewed. They are circulated in order to stimulate discussion and critical comment; any opinions expressed are only those of the author(s).

Copyright LAREFI. All rights reserved. Sections of this material may be reproduced for personal and not-for-profit use without the express written permission of but with acknowledgment to LAREFI. To reproduce the material contained herein for profit or commercial use requires express written permission. To obtain permission, contact LAREFI at cyril.mesmer@u-bordeaux4.fr. 


\section{Sommaire}

1. Identification des canaux de transmission du Quantitative Easing au taux de change .......... 9

2. Analyse empirique des effets globaux du Quantitative Easing sur le taux de change ......... 14

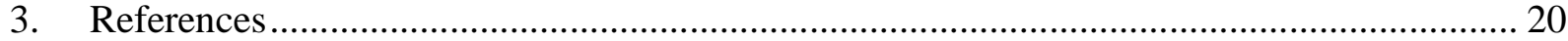




\section{Liste des tableaux}

Tableau 1 Annonce des mesures de Quantitative Easing aux Etats-Unis .................................. 22

Tableau 2 Annonce des mesures de Quantitative Easing au Royaume-Uni ............................... 23

Tableau 3 Annonce des mesures de Quantitative Easing au Japon............................................ 24 


\section{Liste des graphiques}

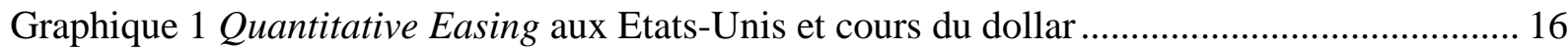

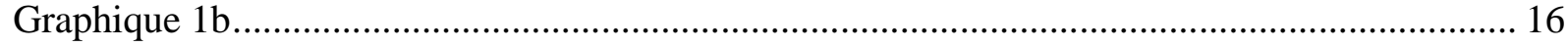




\section{Résumé}

Confrontés au problème de la trappe à liquidité, plusieurs pays industrialisés, dont les EtatsUnis, ont mis en œuvre à partir de 2008 des mesures de Quantitative Easing (QE) afin d'améliorer les conditions de financement dans l'ensemble de l'économie. Bien qu'agissant principalement sur les taux d'intérêt à long terme et les prix des actifs, les politiques de détente quantitative ont également des effets secondaires sur le taux de change. Dans un contexte de "guerre des monnaies ", certains pays industrialisés pourraient alors être tentés d'utiliser le QE comme arme protectionniste afin d'obtenir une dépréciation du taux de change. Après avoir identifié les canaux de transmission du QE au taux de change, cet article propose une analyse empirique basée sur des faits stylisés ainsi que des tests économétriques portant sur les Etats-Unis.

Mots-clés : Quantitative Easing, taux de change, base monétaire, taux d'intérêt à long terme Classification JEL : E44, E51, E52, F31, F41

\section{Abstract}

The effects of Quantitative Easing policies on exchange rate : lessons from american experience

Facing liquidity trap problems, a number of industrialised countries, including the United States and the United Kingdom, have since 2008 implemented Quantitative Easing (QE) measures in an attempt to improve financing conditions in their overall economy. Although Quantitative Easing policies primarily act upon long-term interest rates and asset prices, they also have secondary effects on exchange rates. In a "currency war" context, some countries might be tempted to use QE as a protectionist weapon in a bid to achieve exchange rate depreciation. After identifying which QE transmission channels affect exchange rates, the paper proposes an empirical analysis of stylized facts and econometric tests on the United States. 
Keywords : Quantitative Easing, exchange rate, monetary base, long-term interest rates JEL classification : E44, E51, E52, F31, F41 
Le lancement, jeudi 13 septembre 2012, par la Réserve Fédérale américaine d'un troisième programme d'assouplissement quantitatif (QE3) a ravivé les craintes des principaux partenaires des Etats-Unis, dont principalement la Chine, quant à la volonté de la première puissance mondiale de mettre en place des mesures non conventionnelles de politique monétaire (Quantitative Easing au sens large ${ }^{1}$ ), afin d'obtenir une dépréciation du dollar et donc d'utiliser sa monnaie comme arme commerciale, au risque de déclencher une guerre des monnaies. Comme le souligne P. Artus (2010), la mise en œuvre de politiques monétaires agressives « ne vient pas (probablement) du désir de déprécier le taux de change, mais d'objectifs internes de la politique monétaire (relance du crédit, réduction des taux d'intérêt à long terme, hausse du prix des actifs, soutien de l'inflation anticipée,...) ». En effet, lorsque les taux directeurs sont proches de zéro, comme c'est le cas aux Etats-Unis depuis décembre 2008, l'amélioration des conditions de financement de l'économie ne peut être obtenue que par la mise en place de politiques monétaires non conventionnelles conduisant au gonflement du bilan des banques centrales. Ainsi, la Réserve Fédérale, qui a acheté massivement des titres à long terme depuis la fin 2008 (entre fin 2008 et juin 2011, ces achats se sont élevés à plus de 2000 milliards de dollars), a vu son bilan passer de $6 \%$ du PIB avant la crise financière de 2007-2008 à près de $20 \%$ fin $2011^{2}$. II n'en demeure cependant pas moins vrai que la politique de détente quantitative peut avoir des effets secondaires sur le taux de change qui peuvent s'apparenter à de la protection par le change.

Les Etats-Unis ne sont pas le seul pays à avoir mis en place des politiques monétaires non conventionnelles. Ainsi, la banque d'Angleterre et, plus récemment, la banque centrale du Japon ont également acheté massivement des titre publics, afin de stimuler la croissance et de prévenir la déflation (cf. annexe 1$)^{3}$. Le bilan de la Banque d'Angleterre est ainsi passé de moins de $7 \%$ du PIB avant la crise à près de $24 \%$ en juin 2012. Tout comme les Etats-Unis, l'Angleterre et le Japon pourraient également être 
accusés de manipuler leur taux de change, dès lors que le Quantitative Easing provoquerait une dépréciation de la monnaie nationale.

Alors que de nombreux travaux ont mesuré les effets des politiques de Quantitative Easing sur le prix des actifs et les taux d'intérêt (Gagnon et alii, 2010 ; D’Amico et King, 2010 ; Doh, 2010 et Hamilton et $\mathrm{Wu}, 2010)^{4}$, peu d'études ont été consacrées à la transmission de ces politiques sur le taux de change. La seule étude globale sur le sujet porte sur l'expérience de Quantitative Easing au Japon entre 2001 et 2006 (Terai et alii, 2003) $)^{5}$. Quels sont les principaux canaux de transmission de la politique de Quantitative Easing sur le taux de change ? Quels sont les effets de la politique monétaire agressive aux Etats-Unis sur le dollar ? Les mesures de Quantitative Easing conduisent-elles à une protection par le change ? C'est à ces questions que tente de répondre cet article. Après avoir recensé les principaux canaux d'action du Quantitative Easing sur le taux de change, nous menons une étude empirique sur les Etats-Unis afin d'estimer les effets globaux des politiques récentes de détente quantitative sur le taux de change.

\section{Identification des canaux de transmission du Quantitative Easing au taux de change}

Le Quantitative Easing va agir indirectement sur le taux de change, via ses impacts sur plusieurs autres variables, essentiellement les taux d'intérêt à long terme, la liquidité et le taux d'inflation.

\section{- La transmission par les taux d'intérêt à long terme}

Une politique d'achats par une banque centrale d'actifs financiers à maturité élevée (titres publics à long terme, dettes d'agences, titres adossés à des actifs, obligations d'entreprises) doit provoquer, en théorie, une réduction des taux d'intérêt à long terme, et donc un aplatissement de la courbe des taux d'intérêt. Les mécanismes théoriques sous-jacents sont au nombre de trois. En premier 
lieu, les achats d'actifs par la banque centrale provoquent une baisse de l'offre de titres de long terme à la disposition des investisseurs. Si l'on suppose que la demande de titres de long terme demeure inchangée, l'excès de la demande sur l'offre dû au Quantitative Easing doit entraîner une hausse du prix des actifs à maturité élevée et une baisse des taux d'intérêt à long terme. Deuxièmement, la politique de détente quantitative exerce un effet de signal auprès des agents économiques (Bernanke et alii, 2004). En effectuant une politique d'assouplissement quantitatif, la banque centrale montre en effet sa volonté de maintenir ses taux directeurs à un niveau assez bas, suffisamment longtemps. Si les marchés jugent crédible cet engagement, ils vont anticiper de faibles taux d'intérêt à court terme futurs ${ }^{6}$. Les taux d'intérêt à long terme vont alors baisser, puisque les taux longs reflètent les taux d'intérêt à court terme futurs anticipés. Enfin, en troisième lieu, les achats effectués par la banque centrale exercent des effets bénéfiques sur les marchés financiers lorsque ces derniers sont confrontés à des situations de stress. Ainsi, les écarts entre les taux hypothécaires et les rendements des Bons du Trésor américain ont fortement augmenté lors du pic de la crise financière à la fin 2008, puis ont sensiblement baissé lorsque la FED a annoncé son intention d'acheter des Mortgage Backed Securities (MBS).

Quelles sont les répercussions de la baisse des taux longs sur le taux de change ?

La baisse des taux d'intérêt à long terme dans le pays domestique va s'accompagner d'une réduction du différentiel d'intérêt à long terme entre le pays domestique et l'étranger (on suppose ici que les taux d'intérêt à long terme demeurent inchangés à l'étranger). Conformément à la parité des taux d'intérêt non couverte (PTINC), les marchés doivent alors anticiper une appréciation de la monnaie domestique sur longue période de manière à ce que les actifs domestiques et étrangers offrent la même rentabilité anticipée. Si l'on suppose en outre que le taux de change d'équilibre de long terme demeure inchangé, la monnaie domestique doit se déprécier immédiatement, conformément aux enseignements du modèle de surréaction de Dornbusch (1976). Dans ce modèle, les anticipations de change sont en effet de nature régressive : les anticipations des agents économiques ramènent le taux de change vers 
son niveau de long terme correspondant au taux de change de parité des pouvoirs d'achat (PPA). Selon ce schéma de formation des anticipations de change, les marchés vont anticiper une appréciation du taux de change au comptant futur, si et seulement si le taux de change courant se déprécie davantage que ne l'implique l'équilibre de long terme : il y a donc bien surréaction. Le cadre d'analyse précédent, basé sur la PTINC, est particulièrement adapté pour expliquer la transmission des taux longs au taux de change. En effet, selon les études empiriques les plus récentes (Chinn et Meredith, 2005), la PTINC serait mieux vérifiée à long terme (au-delà d'un an) qu'à court terme.

L'expérience récente des Etats-Unis montre que les politiques de Quantitative Easing ont bien permis de réduire les taux d'intérêt à long terme et ont provoqué une dépréciation du taux de change, conformément aux mécanismes décrits précédemment. Ainsi, l'injection de liquidités par la FED via le programme d'achats de 1725 milliards de dollars de titres longs entre fin 2008 et mars 2010 aurait provoqué une baisse de l'ordre de 50 points de base des taux à long terme (Gagnon et alii, 2010 ; Chung et alii, 2011) ${ }^{7}$. Conformément à la PTINC, l'annonce de ces mesures de détente quantitative a bien provoqué une dépréciation immédiate du dollar (Neelly, 2011). On constate cependant que la dépréciation effective de la monnaie américaine a été inférieure à celle prévue par la PTINC ${ }^{8}$. Ainsi, lorsque la Réserve Fédérale a annoncé, le 18 mars 2009, son intention d’acheter 750 milliards de dollars de plus de MBS, 100 milliards de dollars supplémentaires de dettes d'agences et 300 milliards de dollars d'obligations d'Etat, le dollar aurait dû se déprécier de $6.16 \%$ vis-à-vis de l'euro selon la PTINC, suite à la baisse de 20 points des rendements des bons du Trésor à 10 ans. Dans les faits, la dépréciation n’a été que de $4.93 \%$. Sur la période novembre 2008-mars 2009, l'annonce des mesures de détente quantitative aux Etats-Unis aurait dû provoquer, conformément à la PTINC, une dépréciation cumulée de $16 \%$ du dollar vis-à-vis de l'euro. La dépréciation cumulée enregistrée a été deux fois moindre. Il est à noter que la dépréciation enregistrée peut tenir à des facteurs autres que le Quantitative Easing. L'annonce du QE2 
a également provoqué une dépréciation du dollar via une baisse des taux longs américains, mais on observe que ce canal de transmission a perdu en intensité. Ainsi, selon les résultats des simulations effectuées par Chung et ali (2011), l'impact global du QE2 sur les taux américains à 10 ans ne devait pas excéder - 15 points de base. Les taux longs américains se sont même tendus après la mise en place effective du QE2. Plusieurs explications ont été avancées pour justifier cette hausse (Mufteeva et Julien, 2011). En réagissant par une hausse des taux longs, les marchés ont pu ainsi montrer leur déception visà-vis de la taille du deuxième programme de détente quantitative. Les autorités officielles américaines ont préféré, quant à elles, interpréter cette hausse des taux longs comme le signe d'une amélioration des anticipations des agents sur la croissance américaine. Enfin, la dégradation de la qualité de la dette publique américaine a également pu exercer des pressions à la hausse sur les taux longs américains.

\section{- Les effets de la réallocation des portefeuilles des agents induite par l'augmentation de la liquidité}

Les politiques monétaires non orthodoxes ont pour effet d'accroître les liquidités sur le marché. Si les agents économiques considèrent que les liquidités créées par le Quantitative Easing sont de mauvais substituts des titres acquis par la Banque centrale, ils vont se débarrasser de leurs liquidités excédentaires en achetant d'autres actifs domestiques (actions, immobilier, obligations autres que celles dont on a parlé plus haut) et des actifs étrangers. Il devrait alors s'en suivre des pressions à la hausse sur les prix de ces actifs et des pressions à la baisse sur la monnaie nationale. A la différence des effets mentionnés dans le point précédent, les effets liés à la réallocation des portefeuilles des agents ne se manifestent pas immédiatement. La réallocation des portefeuilles s'effectue en effet à un rythme assez faible.

Comme le souligne P. Artus (2011), l'expérience des Etats-Unis montre cependant que la politique de détente quantitative a conduit assez rapidement à des flux importants de capitaux vers les 
pays émergents (150 milliards de dollars par mois en moyenne), ce qui a provoqué une dépréciation du dollar vis-à-vis des monnaies de ces pays et a conduit ces derniers à « accumuler à nouveau des réserves de change, investies en grande partie en Treasuries pour éviter l'appréciation excessive de leurs devises ». La politique de détente quantitative menée par la banque centrale américaine a donc entraîné une hausse de la base monétaire non seulement aux Etats-Unis, mais également dans les pays émergents.

On constate en outre que l'incidence d'une modification de la politique monétaire américaine sur les flux nets de capitaux vers les pays émergents dépend de l'intensité des liens financiers directs avec les Etats-Unis (FMI, 2011) : un relâchement de la politique monétaire américaine a un plus grand effet positif sur les entrées nettes de capitaux dans les pays qui ont des liens financiers directs intenses avec les Etats-Unis que dans ceux qui n'en ont pas.

\section{- La transmission par le taux d'inflation}

Une injection massive de liquidités peut faire repartir l'inflation anticipée à long terme. Comme le souligne J.P. Betbeze (2010), une politique monétaire trop agressive " pose des problèmes de confiance sur la maîtrise des nouveaux outils ainsi créés par la (banque centrale) et sur sa capacité à les réduire, tandis que l'actif (de la banque centrale) ne cesse de croître ». Si cette politique porte atteinte à la crédibilité de la banque centrale, les agents vont anticiper une augmentation de l'inflation future. L'augmentation de l'inflation importée provoquée par la dépréciation de la monnaie nationale, conformément aux mécanismes mentionnés dans les deux points précédents, va renforcer les anticipations inflationnistes des agents. Finalement, en vertu de la PPA, l'augmentation du différentiel d'inflation anticipé entre le pays domestique et l'étranger, doit conduire les agents à anticiper une dépréciation de la monnaie nationale dans le futur. II convient cependant de mentionner que le risque inflationniste lié à un assouplissement quantitatif est faible tant que l'inflation sous-jacente est sous 
contrôle. Ainsi, l'exemple des Etats-Unis sur la période 2008-2012 montre que la politique monétaire non conventionnelle ne s'accompagne pas d'une augmentation de l'inflation anticipée (Artus, 2012).

En théorie, une politique de détente quantitative doit provoquer, conformément aux mécanismes décrits précédemment, une dépréciation du taux de change. Nous nous proposons de mener une étude empirique pour vérifier l'impact global des mesures récentes de Quantitative Easing sur le taux de change.

\section{Analyse empirique des effets globaux du Quantitative Easing sur le taux de change}

L'étude empirique porte sur les Etats-Unis, premier pays ayant pratiqué le Quantitative Easing après la crise de 2007-2008. Après avoir mis en évidence graphiquement la corrélation entre le taux de change et la base monétaire relative du pays domestique (la base monétaire est retenue comme variable représentative du Quantitative Easing), on estime une équation simple de détermination du taux de change qui incorpore les effets du Quantitative Easing au travers de ceux de la variation de la base monétaire relative du pays domestique.

La corrélation positive entre accroissement de la base monétaire relative du pays domestique par rapport à l'étranger et dépréciation de la monnaie nationale apparaît nettement aux Etats-Unis depuis que la FED a adopté des mesures d'assouplissement quantitatif (graphique 1). Ainsi, le coefficient de corrélation entre croissance de la base monétaire relative des Etats-Unis par rapport à la zone euro et dépréciation du dollar vis-à-vis de la monnaie unique s'est établi à 0.54 entre octobre 2008 et février 2012, contre -0.77 entre janvier 2000 et septembre 2008. Sur la base du taux de change effectif, le coefficient de corrélation entre croissance de la base monétaire relative des Etats-Unis et dépréciation 
du dollar ressort à 0.35 sur la période octobre 2008-février 2012. Toutefois, corrélation ne signifie pas causalité.

Afin d'estimer plus précisément les effets d'une détente quantitative sur le taux de change, nous avons retenu une fonction dérivée du modèle monétaire à prix flexibles. A côté des variables explicatives traditionnelles mises en exergue par ce modèle (différentiel de taux d'intérêt, niveau relatif de l'activité dans le pays domestique par rapport à l'étranger), notre fonction de détermination du taux de change retient la base monétaire relative du pays domestique par rapport à l'étranger comme variable représentative du Quantitative Easing :

$S=f(B M R, D I F I N T, P R O D R)(1)$

où $\mathrm{S}$ représente le taux de change bilatéral (cotation à l'incertain de la monnaie nationale), BMR le ratio base monétaire du pays domestique/base monétaire du pays étranger, DIFINT le différentiel de taux d'intérêt à court terme entre le pays domestique et le pays étranger (les taux d'intérêt à court terme sont mesurés par les taux directeurs), PRODR le ratio production manufacturière domestique/production manufacturière étrangère.

Les variables S, BMR et PRODR sont exprimées en logarithmes.

Comment le taux de change doit-il réagir, en théorie, aux variations des différentes variables explicatives ? Conformément aux mécanismes décrits dans la section précédente, une politique de détente quantitative (i.e. une augmentation de la base monétaire relative du pays domestique) doit provoquer une dépréciation de la monnaie nationale (i.e. une hausse de $\mathrm{S}$ puisque la monnaie nationale est cotée à l'incertain). 


\section{Graphique 1 Quantitative Easing aux Etats-Unis et cours du dollar}

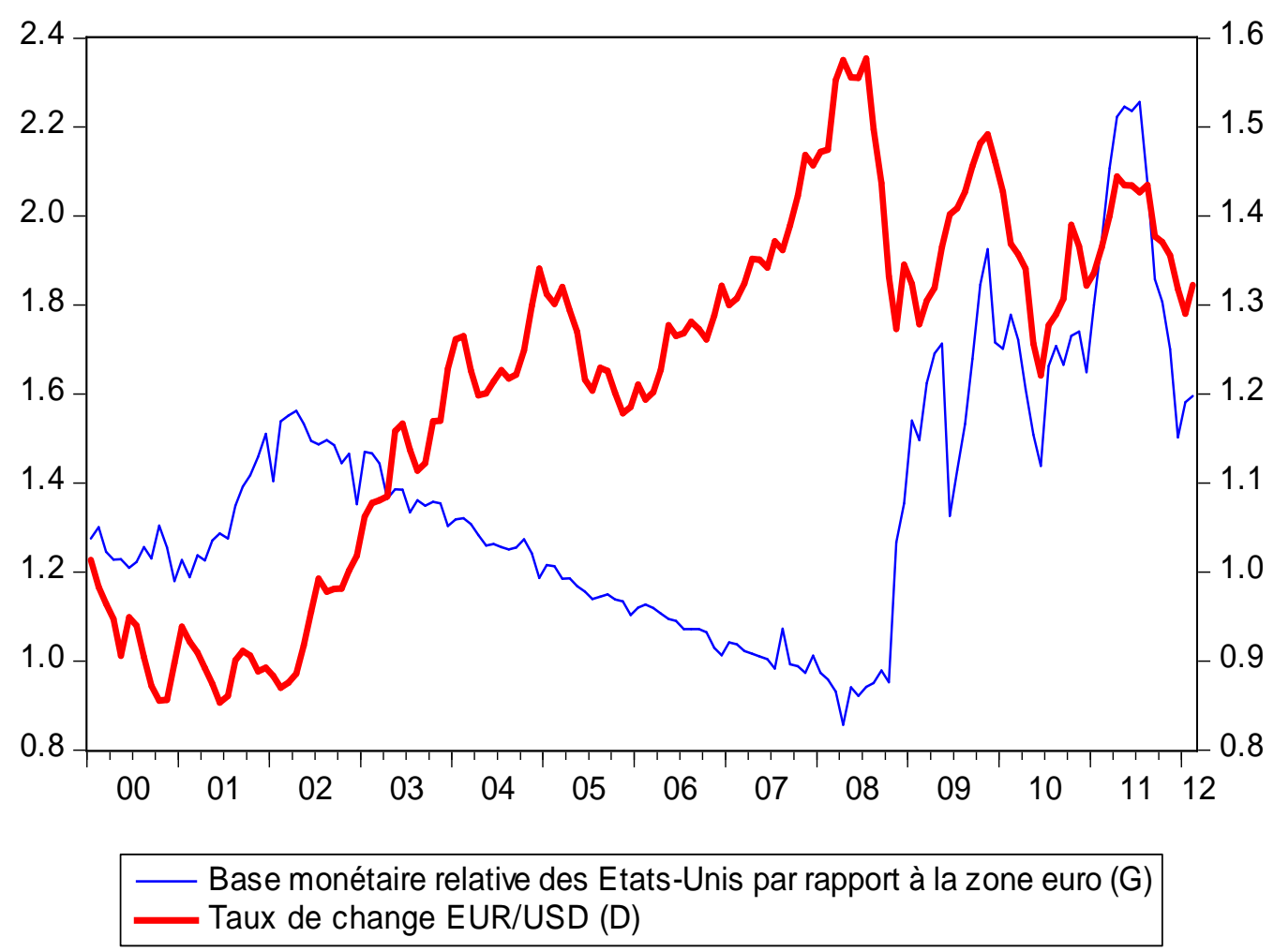

\section{Graphique 1b}

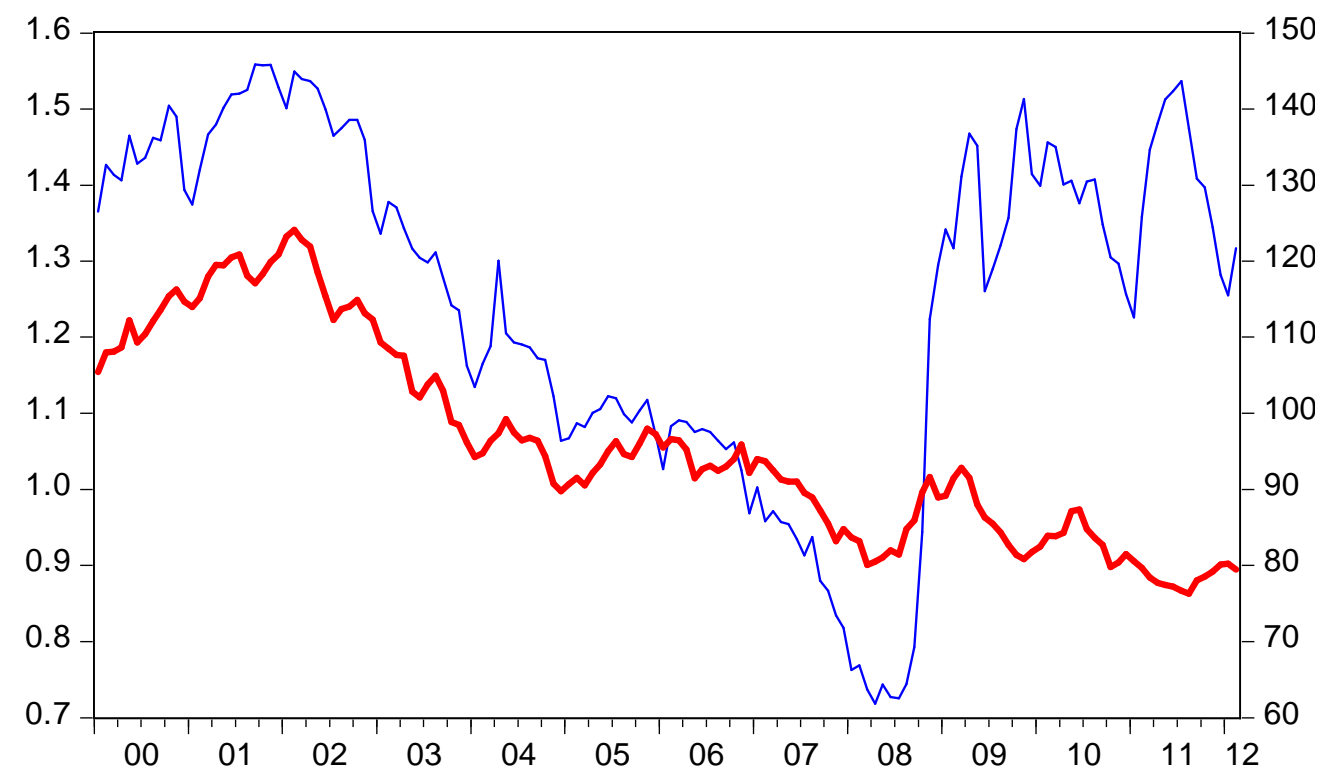

Base monétaire relative des Etats-Unis par rapport à leurs principaux partenaires (G) Taux de change effectif nominal du dollar $(1990=100)(D)$ 
Une variation du différentiel de taux d'intérêt à court terme entre le pays domestique et le pays étranger a, quant à elle, un effet indéterminé sur le taux de change. Dans le cadre du modèle de Mundell-Fleming en économie ouverte, une augmentation du différentiel de taux d'intérêt en faveur du pays domestique induit une appréciation de la monnaie nationale. En revanche, selon le modèle monétaire à prix flexibles, une hausse de ce différentiel d'intérêt doit engendrer une dépréciation de la monnaie nationale puisque la demande relative de monnaie domestique va diminuer. Par ailleurs, une augmentation de la production relative de biens manufacturés domestiques doit provoquer une appréciation de la monnaie nationale. En effet, un pays affichant un taux de croissance supérieur à celui de ses partenaires offre des perspectives d'investissement plus intéressantes, ce qui attire les capitaux étrangers et exerce des pressions haussières sur sa monnaie. Nous avons également estimé les effets de la politique de détente quantitative sur le taux de change effectif nominal du dollar. Pour ce faire, nous avons retenu la fonction suivante $:$ TCE $=f(B M R$, DIFINT, PRODR) (2), où TCE représente le taux de change effectif de la monnaie nationale, BMR la base monétaire relative du pays domestique par rapport à ses partenaires, DIFINT le différentiel de taux d'intérêt à court terme entre le pays domestique et ses partenaires, PROD la production manufacturière relative du pays domestique par rapport à ses partenaires ${ }^{9}$.

Sachant que les taux de change effectifs sont cotés au certain (une hausse de TCE traduit une appréciation de la monnaie nationale), une augmentation de la base monétaire relative du pays domestique doit provoquer une baisse du taux de change effectif. Conformément aux mécanismes décrits précédemment, le taux de change effectif doit dépendre, en outre, positivement de la production manufacturière relative domestique et positivement ou négativement du différentiel de taux d'intérêt.

Les fonctions (1) et (2) ont été estimées sur la période novembre 2008-février 2012 en utilisant la méthode des moindres carrés ordinaires. Nous avons testé au préalable la stationnarité des variables 
retenues. Les résultats des tests (annexe 2 ) montrent que la plupart des variables sont non stationnaires en niveau, d'où la nécessité pour les rendre stationnaires de considérer leurs différences premières. Les variables non stationnaires en niveau ont alors été remplacées dans les fonctions (1) et (2) par leurs différentiels d'ordre 1 stationnaires. Les principaux résultats des régressions sont présentés dans l'annexe 3. Ces résultats montrent que les effets du Quantitative Easing sur le taux de change du dollar diffèrent selon les parités retenues. L'augmentation de la base monétaire relative aux Etats-Unis contribue, de manière significative, dans l'ensemble des spécifications retenues à une dépréciation du dollar, d'une part, vis-à-vis de l'euro et, d'autre part, en termes effectifs ${ }^{10}$. Ainsi, une hausse de $10 \%$ de la base monétaire relative des Etats-Unis par rapport à la zone euro provoque une dépréciation de $1 \%$ du dollar vis-à-vis de l'euro. En termes effectifs, la dépréciation du dollar consécutive à une augmentation de $10 \%$ de la base monétaire relative des Etats-Unis par rapport à leurs partenaires est de $0.5 \%$. En revanche, le Quantitative Easing américain n'a pas d'effet significatif sur les parités dollar/yen et dollar/livre. La parité dollar/livre est fortement influencée par le différentiel d'intérêt entre les Etats-Unis et le Royaume-Uni.

Dans cet article, nous avons essayé, à partir de l'expérience récente des Etats-Unis, de voir si les politiques de Quantitative Easing ont un impact sur le taux de change. Afin d'apporter des éléments de réponse à cette question, dont l'importance est attestée par la présence du thème dans le débat sur la guerre des monnaies, nous avons estimé un modèle simple de détermination du taux de change, qui incorpore les effets des politiques de détente quantitative. Les résultats obtenus permettent de valider la thèse selon laquelle les politiques de Quantitative Easing pourraient être utilisées à des fins protectionnistes, afin d'obtenir une dépréciation du taux de change. Les politiques de détente quantitative (QE1 et QE2) adoptées par les Etats-Unis ont en effet provoqué une dépréciation du dollar, notamment vis-à-vis de l'euro et en termes effectifs, ce qui a permis d'améliorer la compétitivité-prix du 
pays. L'efficacité du Quantitative Easing comme arme protectionniste n'est pas pour autant garanti, dans la mesure où le commerce extérieur américain est peu sensible aux variations du cours du dollar. Par ailleurs, les résultats obtenus montrent que plusieurs parités bilatérales du dollar vis-à-vis des monnaies des principaux partenaires des Etats-Unis n'ont pas été affectées significativement par le Quantitative Easing américain.

Dans ces conditions, plusieurs prolongements doivent être envisagés afin de procéder à une évaluation complète des effets du Quantitative Easing sur le taux de change. Tout d'abord, l'analyse empirique doit être étendue aux autres pays industrialisés qui ont mis en place des mesures non conventionnelles de politique monétaire. Sachant que les taux de change dépendent fortement des anticipations que forment les agents sur l'évolution future des politiques économiques, il est intéressant de voir ensuite comment les taux de change réagissent aux politiques de détente quantitatives lorsque celles-ci sont anticipées par les agents économiques. Enfin, une étude empirique sur des données plus fines (données journalières) doit permettre de préciser les effets des annonces des mesures de Quantitative Easing sur les taux de change. 


\section{References}

ARTUS P. (2012), "La politique monétaire non conventionnelle a-t-elle conduit à une hausse de I'inflation anticipée aux Etats-Unis ?», Natixis, Flash Economie, n 410, 11 juin 2012.

ARTUS P. (2010), " S'il y avait vraiment guerre des monnaies (currency war), qui la gagnerait ? ", Natixis, Flash Economie, $\mathrm{n}^{\circ}$ 620, 18 novembre.

ARTUS P. (2011), "Que faire après le Quantitative Easing 2 ? ", Natixis, Flash Economie, n 111, 11 février.

BERNANKE B.S., REINHART V.R. et SACK B.P. (2004), « Monetary Policy Alternatives at the Zero Bound: an Empirical Assessment", Brookings Papers on Economic Activity, $\mathrm{n}^{\circ} 2$

BETBEZE J.-P. (2010), "Quantitative Easing 2 : les risques", Crédit Agricole SA, ECO News, n 157, 26 octobre.

BLINDER A.S. (2010), "Quantitative Easing: Entrance and Exit Strategies ", FRB of St. Louis Review, novembre-décembre

BOUVERET A., BRAHMI A., KALANTZIS Y., OLMEDO A. et SORBE S. (2009), "Politiques monétaires non conventionnelles : un bilan ", Trésor-Eco, $\mathrm{n}^{\circ} 56$, avril.

CHINN M.D. et MEREDITH G. (2005), "Testing Uncovered Interest Parity at Short and Long Horizons during the Post-Bretton Woods Era", NBER Working paper $n^{\circ} 11077$, January.

CHUNG H., LAFORTE J.P., REIFSCHNEIDER D. et WILLIAMS J.C. (2011),“Have We Underestimated the Likelihood and Severity of Zero Lower Bound Events ?", FRBSF Working Paper, $n^{\circ} 1$.

D'AMICO S. et KING T.B. (2010), "Flow and Stock Effects of Large-Scale Treasury Purchases", Finance and Economics Discussion Series, Federal Reserve Board, $n^{\circ} 52$.

DOH T. (2010), "The Efficacy of Large-Scale Asset Purchases at the Zero Lower Bound", FRB Kansas City Economic Review, Q2.

DORNBUSCH R. (1976), "Expectations and Exchange Rate Dynamics", Journal of Political Economy, November-December. 
Fonds Monétaire International (2011), Perspectives économiques mondiales, chap. 4, avril

GAGNON J., RASKIN M., REMACHE J. et SACK B. (2010), " Large-Scale Asset Purchases by the Federal Reserve : Did They Work ? ", FRBNY Economic Policy Review, mai

HAMILTON J.D. et WU J. (2010), « The Effectiveness of Alternative Monetary Policy Tools in a Zero Lower Bound Environnement », Working paper, University of California of San Diego.

GAVIN W.T. (2009), "More Money : Understanding Recent Changes in the Monetary Base", FRB of St. Louis Review, March/April.

JOYCE M., LASAOSA A., STEVENS I. et TONG M. (2010), " The Financial Market Impact of Quantitative Easing ", Bank of England, Working Paper, $\mathrm{n}^{\circ} 393$.

KOZICKI S., SANTOR E. et SUCHANEK L. (2011), "L'expérience internationale du recours à une mesure de politique monétaire non traditionnelle : l'achat d'actifs par les banques centrales", Revue de la banque du Canada, Printemps.

LOISEL O. et MESONNIER J-S. (2009), « Les mesures non conventionnelles de politique monétaire face à la crise », Banque de France, Questions actuelles, $n^{\circ} 1$.

MUFTEEVA I. et JULIEN T. (2011), "QE2: effectivité des canaux de transmission de la politique monétaire ", Natixis, Flash Economie, n 114, 11 février.

NEELY C.J. (2011), "The Large-Scale Asset Purchases Had Large International Effects", FRB of St. Louis Working Paper Series, $\mathrm{n}^{\circ} 018 \mathrm{C}$.

TERAI A., IIDA Y. et HAMADA K. (2003), "Exchange Rate as Monetary Policy Channel", ESRI Discussion Paper Series, $n^{\circ} 59$

UGAI H. (2007), "Effects of the Quantitative Easing Policy : a Survey of Empirical Analyses", Monetary and Economics Studies, March. 


\section{Annexe 1}

Tableau 1 Annonce des mesures de Quantitative Easing aux Etats-Unis

\begin{tabular}{|c|c|c|}
\hline Date & Décision & Autre information \\
\hline 25 novembre 2008 & $\begin{array}{l}\text { La Fed annonce qu'elle va acheter pour } 100 \\
\text { milliards de dollars de dette GSE (Government } \\
\text { sponsored enterprises) et jusqu'à } 500 \text { milliards de } \\
\text { MBS (Mortgage Backed Securities). }\end{array}$ & \\
\hline $1^{\text {er }}$ décembre 2008 & $\begin{array}{l}\text { Ben Bernanke indique que la Fed pourrait acheter } \\
\text { des Treasuries à long terme. }\end{array}$ & \\
\hline 16 décembre 2008 & $\begin{array}{l}\text { Le communiqué du FOMC mentionne la possibilité } \\
\text { d'acheter des Treasuries à long terme. }\end{array}$ & $\begin{array}{l}\text { Baisse du taux cible des Federal funds de } \\
1 \% \text { à une fourchette entre } 0 \text { et } 0.25 \% \text {. }\end{array}$ \\
\hline 28 janvier 2009 & $\begin{array}{l}\text { Le communiqué du FOMC met l'accent sur la } \\
\text { nécessité de poursuivre les achats de dettes } \\
\text { d'agences et de MBS. Des achats de Treasuries à } \\
\text { long terme sont également envisagés. }\end{array}$ & $\begin{array}{l}\text { Lancement d'une facilité de financement } \\
\text { (la TALF pour Term Asset-Backed } \\
\text { Securities Loan Facility). }\end{array}$ \\
\hline 18 mars 2009 & $\begin{array}{l}\text { La Fed annonce qu'elle va acquérir } 750 \text { milliards } \\
\text { de dollars de plus de MBS, } 100 \text { milliards de dollars } \\
\text { supplémentaires de dettes d'agences et } 300 \\
\text { milliards de dollars de Treasuries à long terme }\end{array}$ & \\
\hline 21 septembre 2010 & $\begin{array}{l}\text { Le FOMC indique qu'un assouplissement } \\
\text { supplémentaire de la politique monétaire est } \\
\text { envisagé pour soutenir la croissance. }\end{array}$ & \\
\hline 3 novembre 2010 & $\begin{array}{l}\text { Le FOMC lance un nouveau programme d'achat de } \\
\text { titres de long terme (QE 2), consistant en l'achat de } \\
600 \text { milliards de dollars de titres du Trésor sur la } \\
\text { période novembre 2010-juin 2011. Le FOMC se } \\
\text { réserve le droit de réviser le rythme d'achat comme } \\
\text { la taille du programme si les circonstances } \\
\text { l'exigent. }\end{array}$ & \\
\hline 9 août 2011 & $\begin{array}{l}\text { Le communiqué du FOMC laisse entrevoir la } \\
\text { possibilité de la mise en place d'un nouveau } \\
\text { programme d'achat de titres de long terme. }\end{array}$ & $\begin{array}{l}\text { La Fed s'est engagée à maintenir le taux } \\
\text { directeur entre } 0 \text { et } 0.25 \% \text { au moins } \\
\text { jusqu'à la mi-2013. }\end{array}$ \\
\hline 21 septembre 2011 & $\begin{array}{l}\text { La Fed lance «l'opération Twist» consistant à } \\
\text { vendre d'ici la fin juin } 2012 \text { pour } 400 \text { milliards de } \\
\text { dollars de bons du Trésor à maturité de } 3 \text { ans et } \\
\text { moins, pour en racheter un montant équivalent } \\
\text { d'une maturité allant de } 6 \text { ans à } 30 \text { ans. }\end{array}$ & $\begin{array}{l}\text { Les taux directeurs restent compris entre } 0 \\
\text { et } 0.25 \% \text {. }\end{array}$ \\
\hline 20 juin 2012 & $\begin{array}{l}\text { Les membres du FOMC ont décidé de prolonger } \\
\text { « l'opération Twist » jusqu'à la fin décembre } 2012 \text {. } \\
\text { La FED achètera encore pour } 267 \text { milliards de } \\
\text { dollars de Treasuries à long terme, en vendant un } \\
\text { montant équivalent de titres courts, afin de laisser } \\
\text { inchangée la taille de son bilan }\end{array}$ & \\
\hline 13 septembre 2012 & $\begin{array}{l}\text { La Fed annonce un troisième programme } \\
\text { d'assouplissement quantitatif (QE3). Ce plan } \\
\text { consistera à racheter chaque mois } 40 \text { milliards de } \\
\text { dollars de MBS et à poursuivre les rachats de titres } \\
\text { sur les marchés }\end{array}$ & $\begin{array}{l}\text { Les taux directeurs seront maintenus entre } \\
0 \text { et } 0.25 \% \text { au moins jusqu'à la mi } 2015\end{array}$ \\
\hline
\end{tabular}

Source : C.J. Neely (2010) + actualisation auteur 
Tableau 2 Annonce des mesures de Quantitative Easing au Royaume-Uni

\begin{tabular}{|c|c|c|}
\hline Date & Décision & Autre information \\
\hline 11 février 2009 & $\begin{array}{l}\text { Le Rapport sur l'inflation du mois de } \\
\text { février et la conférence de presse associée } \\
\text { ont laissé entendre que des achats de titres } \\
\text { étaient possibles. }\end{array}$ & \\
\hline 5 mars 2009 & $\begin{array}{l}\text { Le Comité de politique monétaire (MPC) } \\
\text { annonce qu'il pourrait acheter jusqu'à } 75 \\
\text { milliards de livres d'actifs dans les trois } \\
\text { prochains mois. Ces achats seraient } \\
\text { financés par création monétaire à travers } \\
\text { une augmentation des réserves des } \\
\text { banques commerciales. Concernant les } \\
\text { titres publics, les achats de la BoE } \\
\text { porteront uniquement sur les GILTS ayant } \\
\text { une maturité résiduelle comprise entre } 5 \\
\text { et } 25 \text { ans }\end{array}$ & $\begin{array}{l}\text { Les taux directeurs } \\
\text { passent de } 1 \% \text { à } 0.5 \% \text {. }\end{array}$ \\
\hline 7 mai 2009 & $\begin{array}{l}\text { Le MPC annonce que le montant des } \\
\text { achats d'actifs est porté à } 125 \text { milliards de } \\
\text { livres }\end{array}$ & \\
\hline 6 août 2009 & $\begin{array}{l}\text { Le MPC annonce que le montant des } \\
\text { achats d'actifs est porté à } 175 \text { milliards de } \\
\text { livres. Désormais, la BoE peut acheter des } \\
\text { GILTS dont la maturité résiduelle est } \\
\text { supérieure à } 3 \text { ans. }\end{array}$ & \\
\hline 4 février 2010 & $\begin{array}{l}\text { Le MPC annonce que le montant des } \\
\text { achats d'actifs devrait être fixé à } 200 \\
\text { milliards de livres }\end{array}$ & $\begin{array}{l}\text { Le MPC se réserve le } \\
\text { droit de moduler le } \\
\text { programme d'achats } \\
\text { d'actifs }\end{array}$ \\
\hline 6 octobre 2011 & $\begin{array}{l}\text { Le MPC annonce que le montant des } \\
\text { achats d'actifs est porté à } 275 \text { milliards de } \\
\text { livres. Ce nouveau programme d'achat } \\
\text { d'actifs sera mis en ouvre au cours des } \\
\text { quatre prochains mois }\end{array}$ & $\begin{array}{l}\text { Le volume du } \\
\text { programme d'achat } \\
\text { d'actifs pourrait être } \\
\text { ajusté à la hausse. } \\
\text { Les taux directeurs sont } \\
\text { maintenus au niveau de } \\
0.5 \%\end{array}$ \\
\hline 9 février 2012 & $\begin{array}{l}\text { Le MPC annonce un relèvement du } \\
\text { plafond d'achats d'actifs de } 275 \text { milliards } \\
\text { de livres à } 325 \text { milliards de livres }\end{array}$ & \\
\hline 5 juillet 2012 & $\begin{array}{l}\text { Le MPC décide de relever le plafond de } \\
\text { ses achats d'actifs de } 325 \text { milliards de } \\
\text { livres à } 375 \text { milliards }\end{array}$ & \\
\hline
\end{tabular}

Source : Joyce et alii (2010) + actualisation auteur 
Tableau 3 Annonce des mesures de Quantitative Easing au Japon

\begin{tabular}{|c|c|c|}
\hline Date & Décision & Autre information \\
\hline 5 octobre 2010 & $\begin{array}{l}\text { Le Comité de Politique monétaire de la } \\
\text { BdJ annonce la création d'un fonds de } \\
5000 \text { milliards de yens destiné à financer } \\
\text { des achats d'actifs (emprunts d'Etat, } \\
\text { billets de Trésorerie, obligations } \\
\text { d'entreprises) }\end{array}$ & $\begin{array}{l}\text { La BdJ annonce une } \\
\text { baisse de son taux } \\
\text { directeur de } 0 \text { à } 0.1 \% \text {, } \\
\text { contre } 0.1 \% \text { depuis } \\
\text { décembre } 2008 .\end{array}$ \\
\hline 23 octobre 2010 & $\begin{array}{l}\text { La BdJ adopte un programme d'achat } \\
\text { d'actifs financiers de } 5000 \text { milliards de } \\
\text { yens, se décomposant de la façon } \\
\text { suivante : } \\
\text {-achat d'obligations d'Etat et de bons du } \\
\text { Trésor à court terme pour un montant de } \\
3500 \text { milliards de yens } \\
\text {-achat de billets de trésorerie pour un } \\
\text { montant de } 500 \text { milliards de yens } \\
\text {-achat de d'obligations d'entreprises pour } \\
\text { un montant de } 500 \text { milliards de yens } \\
\text {-achat d'actifs risqués (fonds de } \\
\text { placements immobiliers et fonds négociés } \\
\text { en bourse) pour un montant de } 500 \\
\text { milliards de yens }\end{array}$ & \\
\hline 14 mars 2011 & $\begin{array}{l}\text { Suite au tremblement terre et au séisme, } \\
\text { la BdJ annonce un accroissement de } 5000 \\
\text { milliards de yens de son programme } \\
\text { d'achats d'actifs, qui pourrait être étendu } \\
\text { si les circonstances l'exigent. }\end{array}$ & \\
\hline 4 août 2011 & $\begin{array}{l}\text { La BdJ a étendu de } 5000 \text { milliards de } \\
\text { yens son programme d'achat d'actifs. Au } \\
\text { total, ce programme s'élève à } 15000 \\
\text { milliards de yens }\end{array}$ & \\
\hline 14 février 2012 & $\begin{array}{l}\text { La BdJ annonce l'achat de } 10000 \\
\text { milliards de yens d'obligations d'Etat } \\
\text { supplémentaires d'ici à la fin de } 2012 \text {. } \\
\text { Cette décision porte à } 65000 \text { milliards de } \\
\text { yens son programme d'achats d'actifs }\end{array}$ & \\
\hline 19 septembre 2012 & $\begin{array}{l}\text { La BdJ annonce que le programme } \\
\text { d'achat d'actifs sera augmenté de } 10000 \\
\text { milliards de yens à } 80000 \text { milliards de } \\
\text { yens d'ici à décembre } 2013\end{array}$ & \\
\hline
\end{tabular}

Source : Auteur 


\section{Annexe 2 Tests de racine unitaire}

Pour tester la stationnarité des variables, nous avons utilisé le test de Dickey et Fuller augmenté (ADF) et le test de Philips et Perron. Tous les tests ont été effectués au seuil de $1 \%$. Les résultats de ces tests sont présentés dans le tableau suivant :

\begin{tabular}{|c|c|c|c|c|}
\hline \multirow[t]{2}{*}{ Variables } & \multicolumn{2}{|c|}{$\mathrm{ADF}$} & \multicolumn{2}{|c|}{ Philips et Perron } \\
\hline & Niveau & $\begin{array}{r}\text { Différences } \\
\text { premières }\end{array}$ & Niveau & $\begin{array}{l}\text { Différences } \\
\text { premières }\end{array}$ \\
\hline Taux de change USD/EUR & -3.41 & $-4.78 *$ & -2.55 & $-5.30 *$ \\
\hline Taux de change USD/YEN & $-4.24 *$ & $-4.95 *$ & -3.56 & $-5.11 *$ \\
\hline Taux de change USD/GBP & -3.63 & $-4.18 *$ & -3.79 & $-4.18 *$ \\
\hline TCE du dollar & -3.44 & $-4.77 *$ & -2.58 & $-4.67 *$ \\
\hline BMR des Etats-Unis par rapport à la zone euro & -2.45 & $-5.89 *$ & -2.45 & $-5.92 *$ \\
\hline BMR des Etats-Unis par rapport au Japon & $-6.72 *$ & $-6.55^{*}$ & $-6.59 *$ & $-5.79 *$ \\
\hline $\begin{array}{l}\text { BMR des Etats-Unis par rapport au Royaume- } \\
\text { Uni }\end{array}$ & $-5.01 *$ & $-5.91 *$ & -2.11 & $-5.96 *$ \\
\hline $\begin{array}{l}\text { BMR des Etats-Unis par rapport à leurs } \\
\text { partenaires }\end{array}$ & -3.17 & $-4.22 *$ & $-5.42 *$ & $-4.27 *$ \\
\hline DIFINT entre les Etats-Unis et la zone euro & -3.56 & $-8.56^{*}$ & -1.92 & $-7.85^{*}$ \\
\hline DIFINT entre les Etats-Unis et le Japon & $-12.21 *$ & $-12.81 *$ & $-39.07 *$ & $-12.81 *$ \\
\hline DIFINT entre les Etats-Unis et le Royaume-Uni & ---- & ---- & -3.94 & $-7.81 *$ \\
\hline $\begin{array}{l}\text { DIFINT entre les Etats-Unis et leurs principaux } \\
\text { partenaires }\end{array}$ & -1.78 & $-9.79 *$ & -1.75 & $-8.39 *$ \\
\hline PRODR entre les Etats-Unis et la zone euro & -0.20 & $-6.32 *$ & -1.46 & $-6.32 *$ \\
\hline PRODR entre les Etats-Unis et le Japon & -2.19 & $-4.98 *$ & -2.00 & $-5.06^{*}$ \\
\hline PRODR entre les Etats-Unis et le Royaume-Uni & -1.65 & $-6.26^{*}$ & -1.68 & $-6.64 *$ \\
\hline $\begin{array}{l}\text { PRODR entre les Etats-Unis et leurs principaux } \\
\text { partenaires }\end{array}$ & -2.44 & $-9.25^{*}$ & -2.05 & $-9.45^{*}$ \\
\hline
\end{tabular}

Note :

Toutes les variables, à l'exception du différentiel d'intérêt, sont exprimées en logarithmes.

La régression auxiliaire est effectuée avec une constante et une tendance.

Lorsque les deux tests donnent des résultats contradictoires, on privilégie le test de Philips et Perron dans la mesure où ce test est généralement considéré comme plus performant.

*indique que l'hypothèse nulle (la non stationnarité) est rejetée au seuil de 


\section{Annexe 3 Les effets du Quantitative Easing américain sur le cours du dollar}

\begin{tabular}{|c|c|c|c|c|c|c|c|c|c|c|}
\hline Parités & Constante & $\mathrm{BMR}_{-1}$ & $\Delta\left(\mathrm{BMR}_{-1}\right)$ & DIFINT & $\Delta$ (DIFINT) & PRODR & $\Delta(\mathrm{PROD})$ & $\mathbf{R}^{2}$ & DW & $F$ \\
\hline $\begin{array}{c}\Delta(\mathrm{USD} / \mathrm{EUR}) \\
(1 \mathrm{EUR}=\mathrm{S} . \mathrm{USD})\end{array}$ & $\begin{array}{l}0.00067 \\
(0.0044)\end{array}$ & & $\begin{array}{l}0.103 \\
(0.047) * *\end{array}$ & & $\begin{array}{l}-0.013 \\
(0.026)\end{array}$ & & $\begin{array}{l}-0.689 \\
(0.389) *\end{array}$ & 0.22 & $1.81^{\mathrm{a}}$ & $3.42 * *$ \\
\hline $\begin{array}{l}\Delta(\mathrm{USD} / \mathrm{YEN}) \\
(1 \mathrm{YEN}=\mathrm{S} . \mathrm{USD})\end{array}$ & $\begin{array}{l}-0.134 \\
(0.116)\end{array}$ & $\begin{array}{l}-0.034 \\
(0.031)\end{array}$ & & $\begin{array}{l}0.040 \\
(0.046)\end{array}$ & & & $\begin{array}{c}0.056 \\
(0.085)\end{array}$ & 0.17 & $1.64^{\mathrm{a}}$ & $2.61 *$ \\
\hline $\begin{array}{l}\Delta(\mathrm{USD} / \mathrm{GBP}) \\
(1 \mathrm{GBP}=\mathrm{S} . \mathrm{USD})\end{array}$ & $\begin{array}{l}0.0030 \\
(0.0039)\end{array}$ & & $\begin{array}{r}-0.039 \\
(0.030)\end{array}$ & & $\begin{array}{l}-0.058 \\
(0.014)^{* * *}\end{array}$ & & $\begin{array}{l}-0.023 \\
(0.369)\end{array}$ & 0.37 & $1.77^{\mathrm{a}}$ & $7.14 * * *$ \\
\hline $\begin{array}{l}\Delta(\text { TCEUSD }) \\
(1990=100)\end{array}$ & $\begin{array}{c}0.012 \\
(0.0086)\end{array}$ & $\begin{array}{c}-0.052 \\
(0.026)^{*}\end{array}$ & & & $\begin{array}{l}-0.002 \\
(0.024)\end{array}$ & & $\begin{array}{l}0.190 \\
(0.225)\end{array}$ & 0.18 & $1.32^{\mathrm{b}}$ & $2.10 *$ \\
\hline
\end{tabular}

Note : Toutes les variables, à l'exception du différentiel d'intérêt, sont exprimées en logarithmes.

Les problèmes d'hétéroscédasticité ont été traités en retenant la procédure proposée par White.

Les valeurs entre parenthèses représentent les écarts-types estimés.

$*, * *, * * *$ : significatifs à $10 \%, 5 \%, 1 \%$.

${ }^{a}$ : Absence d'autocorrélation des erreurs ${ }^{b}$ :La valeur de DW ne permet pas de tirer de conclusion quant à l'autocorrélation des erreurs 


\section{RECENT LAREFI Working PAPERS}

For earlier LAREFI Working Papers, please go to http://lare-efi.u-bordeaux4.fr

All Discussion Papers can be downloaded free of charge

CR13-EFI/01 Bouet, A., Estradres, C., Laborde, D., "Households heterogeneity in a global CGE model : an illustration with the MIRAGE-HH (MIRAGE-HouseHolds) model»

CR12-EFI/05 Chiappini, R., , "Les indices composites sont-ils de bonnes mesures de la compétitivité des pays ?"

CR12-EFI/04 Chiappini, R., , "Un réexamen de la relation entre commerce et Investissement Direct à l'étranger (IDE) à partir d'un modèle en panel dynamique. Le cas de l'Allemagne, la France et l'Italie"

CR12-EFI/03 Brana, S., Djibenou, M-L., Prat, S., "Global excess liquidity and asset prices in emerging countries : a pvar approach"

CR12-GED/171 Roca T., "A Methodology for Comparing Governance Database, Institutional Profiles Database Robustness. An example using corruption data, from simple graph representation to advanced econometrics"

CR12-EFI/02 Musson A., "Construire l'attractivité durable régionale : l'exemple de l'Aquitaine"

CR12-EFI/01 Dupuy L., "International Trade and Sustainability : A survey" 
${ }^{1}$ La notion de Quantitative Easing (QE) désigne plusieurs types de mesures non conventionnelles. Selon la typologie établie par B. Bernanke et ali (2004), on trouve, d'une part, des politiques tournées vers le passif (ie des mesures visant à augmenter la taille du passif de la banque centrale et donc de la base monétaire) et, d'autre part, des politiques tournées vers l'actif (ie des mesures visant à modifier l'actif de la banque centrale, soit par une modification de la maturité des actifs, soit par une modification de la nature des actifs). Le Quantitative Easing pur correspond à la création de base monétaire par achat de titres publics.

${ }^{2}$ La politique non conventionnelle de la FED a pris plusieurs formes: après avoir pratiqué une politique de refinancement des institutions financières, la banque centrale américaine a réalisé des achats massifs de titres publics et de MBS (Mortgage Backed Securities). Entre fin 2008 et mars 2010, la FED a acheté, dans le cadre du QE1, pour 1720 milliards de titres de long terme (1250 milliards en MBS, 300 milliards en Treasuries et 170 milliards en titres de dettes par les agences féférales). Fin novembre 2010, elle a annoncé son intention d'effectuer des achats supplémentaires de titres publics à long terme pour un montant de 600 milliards de dollars (QE2). Le QE2 s'est achevé en juin 2011. Le QE3 a été lancé le 13 septembre 2012. L'annexe 1 présente I'ensemble des mesures de Quantitative Easing adoptées par les Etats-Unis.

${ }^{3}$ La BCE a également mis en œuvre des mesures non conventionnelles, mais celles-ci furent principalement destinées à fournir des liquidités aux banques. Plus récemment, la BCE a adopté plusieurs mesures afin de répondre à la crise des dettes souveraines dans la zone euro.

${ }^{4}$ Kozicki et ali (2011) effectuent un survey des études récentes ayant évalué l'incidence des politiques monétaires non traditionnelles. Les auteurs considèrent que les résultats de ces études tendent à confirmer que les achats massifs d'actifs effectués par la Réserve fédérale américaine et la Banque d'Angleterre ont eu une influence notable sur les marchés financiers.

${ }^{5}$ Les résultats de cette étude montrent que la base monétaire japonaise relative à celle des Etats-unis a un effet significatif sur le taux de change nominal du yen vis-à-vis du dollar.

${ }^{6}$ Si la banque centrale ne respectait pas cet engagement, elle s'exposerait à un risque important de perte en capital puisqu'elle a " chargé " son bilan en titres à long terme (Loisel et Mésonnier, 2009).

${ }^{7}$ Selon Chung et alii (2011), les taux directeurs américains auraient dû diminuer de 200 points de base pour provoquer une baisse équivalente de 50 points de base des rendements des bons du Trésor à 10 ans.

8 La politique de Quantitative Easing mise en œuvre par le Royaume-Uni a également provoqué une baisse des taux longs et une dépréciation de la livre. Selon l'étude réalisée par Joyce et alii (2010), les taux de rendement des GILTS auraient baissé en moyenne de 100 points de base suite à l'annonce des mesures de détente quantitative (cf annexe 1). Compte tenu de cette baisse des taux d'intérêt, les auteurs considèrent, en s'appuyant sur la PTINC, que la livre aurait dû se déprécier de $8 \%$. En effectuant la somme des variations du taux de change qui ont suivi l'annonce des mesures d'assouplissement quantitatif, la dépréciation effective de la livre ressort seulement à $4 \%$. Comme dans le cas américain, on constate donc que le sens de la variation du taux de change faisant suite aux mesures de Quantitative Easing est conforme à ce qui est prévu par la relation de PTINC, mais l'ampleur de la variation constatée est inférieure à celle prédite par cette relation.

${ }^{9}$ Les variables étrangères ont été construites en retenant des coefficients de pondération basés sur la part de chaque partenaire des Etats-Unis dans l'ensemble des échanges commerciaux (exportations et importations) effectués par les Etats-Unis. Les principaux partenaires commerciaux des Etats-Unis sont la zone euro, le Canada, le Royaume-Uni, la Chine et le Japon.

${ }^{10}$ La base monétaire relative des Etats-Unis est retardée d'une période. 